\title{
Gestosis: Ophthalmologic Peculiarities
}

\author{
Galina Bratko $^{1}$, Ekaterina Pichikova ${ }^{1, *}$, Aleksandr Trunov ${ }^{2}$, Valery Chernykh ${ }^{1}$ \\ ${ }^{1}$ Novosibirsk Branch Federal State Institution, “Intersectoral Research and Technology Complex” “Eye microsurgery”, Novosibirsk, Russia \\ ${ }^{2}$ Scientific Center of Clinical and Experimental Medicine, Siberian Branch of the Russian Academy of Medical Sciences, Novosibirsk, \\ Russia \\ *Corresponding author: katiapichikova@mail.ru
}

Received January 30, 2014; Revised February 17, 2014; Accepted February 24, 2014

\begin{abstract}
The most interesting studies of perfusion-diffusion deficiency in the morpho-functional system motherplacenta-fetus available in women with pre-eclampsia and eclampsia are just described in this review. The main cause of vascular endothelium damage is vasospasm and an accelerated formation of free radicals in women's with pre-eclampsia. The ophthalmological peculiarities mentioned in the article are such as follows: irregular spasm of small arterioles, including normal pregnancy cases, is usually described with a method of conjunctive vessels biomicroscopy. The most common method of ophthalmological examination is ophthalmoscopy. Pathologic changes of retina and retinal vessels in women with gestosis are most similar to the changes observed in the eyes of patients with essential hypertension. Pregnant women are in a group of risk because of arterial hypo- and hypertension, anemia and gestosis, providing the expressed deficiency of blood circulation of eye.
\end{abstract}

Keywords: gestosis, myopia, retinal vessels, pregnant women, hemorrhage, retinal detachment

Cite This Article: Galina Bratko, Ekaterina Pichikova, Aleksandr Trunov, and Valery Chernykh, "Gestosis: Ophthalmologic Peculiarities.” American Journal of Medical Sciences and Medicine, vol. 2, no. 1 (2014): 25-28. doi: 10.12691/ajmsm-2-1-6.

\section{Introduction}

The late gestosis is sure to be considered one of the most important topics of the modern obstetrics, laying down in the foundation of the maternal and prenatal mortality structure. There no common theory of pathogenesis and etiology of gestosis nowadays. It makes hampering timely diagnostics, valuation of severity of the disease and preventive measures to be hampered, causing the development and progression of complications. The gestosis is considered to be one of the clinical presentations of an inadequate mother's organism adaptation mechanisms for the further providing fetation. Such pathological conditions are defined with the immunological and autoimmune abnormalities, according to Serov V.N. et al (2002) first and foremost of all. Gestosis is based on SIRS [1]. Meanwhile, a great number of eye pathology of pregnant women is considered to be insufficiently explored by ophthalmologists. The proper solution can be obtained by the cooperation of obstetrician and ophthalmologist, taking into account the development of pathogenic mechanisms of gestosis. These review is intended for an audience of ophthalmologists. We tried to do our best to analyze the present literary data of pathogenesis peculiarities of pregnant women gestosis.

\section{Main Text}

The main point of the article is to analyze the up to day literature data of medical research, concerning some pathological mechanisms of gestosis development and the manifestation of the pathological process, in the purpose of the further development of new methods of diagnoses and treatment.

Gestosis of pregnant women is defined with a pathological condition, arising only in a case of pregnancy, causing development of complications and frequently ending after labors.

It has been known quite long since that embryo is no less than exotoxins (an old wrong name was toxicosis).

Early and late gestosis are distinguished from each other nowadays. The gestosis of the first and second trimester of pregnancy are known to be revealed among women with compromised history. Also, this is an absence of somatic pathology, when the pregnancy is a trigger factor which causes the wrecking of adaptation mechanisms resulting in pregnancy complications.

The early gestosis of pregnant women is revealed in 50$60 \%$ (more often on the 6-12 week of pregnancy); they do need to be corrected in $10 \%$ of cases. Early gestosis of pregnant women are characterized by dyspepsia, vomit, hypersalivation; there are some rare forms of it: dermatitis of pregnancy, bronchial asthma of pregnant women, hepatosis, and even fatty hepatosis of pregnancy. The vomit may cause dehydration and the loss of electrolytes, depression of functional activity of rens (depression of glomerular filtration rate, diurnal diuresis). The plasma concentration of potassium and magnesium is gradually increasing because of the depression of extracellular fluid volume, causing the hypoproteinemia. In this case fat becomes the main source of alimentation, ketone bodies are accumulating, the lipolysis speed increases. Free fatty 
acids transform into ketone bodies in hepar, causing hyperlipidemia of a hard stage up to fatty hepar infiltration. Consequently, ketoacidosis intensifies vomit bringing about the further water and electrolyte imbalance. Extracellular and intracellular fluid emaciation causes a hypoxia of tissues and organs. Hematocrit is increasing because of dehydration which is inadequate to the amount of erythrocytes and hemoglobin, resulting in serious stages of gestosis. In a case of a severe vomit the conjuntival and retinal hemorrhages can happen and are considered as emergency in obstetrics department, even may lead to therapeutic abortion, in case of:

- A grave general condition

- Ineffectiveness of therapy in recent 6-12 hours

- Aggressive clinical behavior of the acute yellow atrophy of liver

- Aggressive clinical behavior of severe hepatic failure

So far as we are concerned with severe gestosis, I'll not be mistaken by saying that the term $\mathrm{OPH}$ is characterized with edema, proteinuria, hypertension.

Gestosis is a type of the pregnancy hypertension frequently appearing in 6-12\% cases of healthy pregnant women and in 20-40\% of women with extragenital diseases [2].

The elevation of blood pressure level precedes the hematocrit elevation. The thrombocytopenia is considered to be one of the symptoms of severe gestosis, preceding the $\mathrm{OPH}$ symptoms. The uteroplacental hemodynamics disturbance can be noted with an approach of Doppler ultrasonography, starting from the 16 week of pregnancy [3]. Women with a high risk of gestosis have a higher average blood pressure. The reologic blood properties disturbance (elevation of blood viscosity) can be notice from the 20-th week of pregnancy, the matter is that the clinical picture may appear only from the 32-36 week of pregnancy.

The groups of patients with a risk of OPH gestosis are:

- The women with renal disorders

- The women with cardio-vascular disorders, essential hypertention, neurocirculatory dystonia, valvular defect.

- The women with endocrinopathy - obesity and diabetes mellitus first and foremost of all.

The frequency of pregnancy and labor complicated with gestosis vary from $10 \%$ to $15 \%$. The part of severe gestosis in the structure of maternal mortality is $12 \%$, while in developing countries is $30 \%$ [5]. The part of severe gestosis in the structure of maternal mortality reaches the value of $20 \%$ in Russian Federation and takes the second place after obstetric hemorrhage $[6,7]$.

\subsection{Some Peculiarities of Late Gestosis}

Pathological mechanisms laying down the foundation of the late gestosis aren't revealed up to now, but endothelium-vascular abnormality is a bottom line of the common processes in placenta, renas end brain [8,9,10,11].

The blood vessels of mothers with preeclampsia are characterized with high capillary permeability with an excessive physical response to angiotensin II [12]. There exists a high receptivity of vessels to both circulating adrenaline and noradrenaline. There is a decrease of receptivity to angiotensin II in a case of a healthy pregnant women, but during gestosis there is a high receptivity to it
[13]. Meanwhile the arteriolespasm causes a high resistance of peripheral vessels and hypertension. Pregnancy is one of the factors of hemodynamic and metabolic body burden and is related to the necessity of the oxygen delivery and sufficient state of fetus hemodynamics [14]. It depends on circulated blood redistribution as a reaction for the increase of placental circulation; as well as there is a corresponding decrease of peripheral circulation in eyes. Physiological blood vessels dilatation and vascular blood resistance are known to happen in a case of a noncomplicated pregnancy and are defined with a sufficient production of equal volumes of vasodilator such as prostacyclin and vasoconstrictive agent such as thromboxane, causing angiospasm and hypertension in the long run. An increased level of the factor VIIIR, fibronectin, protease are considered to be a marker of the endothelial cell disturbance.

No wonder, a rapid free radical production in a state of preeclampsia may cause the destruction of the structure functional integrity of endothelial cells.

Oxidative stress first of all plays the important part in gestosis development $[15,16]$. An activation of lipid peroxidation and a phospholipase A2 is one of the damaging factors of a lipid layer. A tissue hypoxia is a factor of alteration in conditions of depression of an alphamacroglobulin and antioxidant activity as well. The presence of the oxidized thiolic groups $(\mathrm{SH})$ in proteins being included in the inner mitochondrial membrane structure mainly increases membrane permeability. There is a great amount of a literary data, bearing witness to the importance of oxidative stress in gestosis pathogenesis. There is a deficiency of antioxidants, hypoalbuminemia, hypertriglyceridemia, hyperlipoproteinemia practically in every case of gestosis [17]. All the disturbances, mentioned above, are correlated by a severity of the clinical picture of gestosis. Endothelial cell disturbance and a total arteriospasm are very well known to be guiding factors causing systemic disorders and different clinical picture of the disease nowadays. Vasospasm is considered to be the main reason of the endothelial cell disturbance, causing a blood platelet aggregation, decrease of the prostoprostacyclin production, changes in microcirculation and a multiple organ failure. It deals dealing with proteinuria, changes in brain functions and hepar functions, in the ultimate end. There exist some forcible facts, bearing witness to the alteration of a nervous system such as headache, visual disorders [18], hyperreflexia and seizures are among them. Probably, the stroke is the main reason of such disease manifestation, making the contribution to maternal mortality level in case of gestosis. The systemic arteriolospasm causes the decrease of blood circulation volume and tissue edema to some extent.

\subsection{Ophthalmological Peculiarities of Gestosis}

By the aid of biomicroscopy of conjunctive vessels, one can reveal uneven spasm of small arterioles, significant slow-down of a blood flow, in a case of an uncomplicated pregnancy. This is a certain method of an early detection of gestosis because the further progression of this symptom occurs only in a case of a late gestosis. The conjunctive vessels spasm is more sufficient than in retinal vessels even in severe stage of gestosis. 
But the basic method of eye examination is ophthalmoscopy, dealing with a capacity of retina and retinal vessels examination in dynamic. The classification of eye fundus changes can be based on this method of examination. Pathological changes of retinal vessels and retina itself have just the same main features as in case of essential hypertension. To be more precise, it may be characterized by a dilatation and hyperemia of retinal vessels, plethoric retinal arteries, a reflex stripe is wider in particular. The veins are dilated, small vessels become visible and the fundus of eye seemed to be hyperemic as well. The disc of optic nerve is also hyperemic because of the overfilling of retinal vessels.

Myopia frequency forms $18-19 \%$ of the total extragenital pathology. The research conducted in MRSRIOG with a help of SRI of eye diseases by Gelmgolts revealed that myopia doesn't influence on visual functions to a great extent [19].

The removal of the period of spontaneous expulsive efforts is one of the common methods of the prevention of retinal detachment. The baseless increase of the cesarean section frequency, on the one hand, leads to the increase of drawbacks of surgery and to increase of the amount of women of childbearing age with an uterine scar on the other. All the more so, such women are in a state of anxiety and apprehensions of the repetition of cesarean section.

Unfortunately, there isn't any common opinion of the selection criterion of the optimal type of delivery in women with myopia in a case of normal pregnancy and in women with gestosis as well.

Labor per vias naturals in the case of hypertension are fraught with grate loading and changes of general hydrodynamics in the labor period, in the second act of delivery in particular. During this period there are significant changes in cardiovascular system: general peripheral resistance increases in 30\%, the mean dynamic pressure increases in $14 \%$, cerebral blood flow exceeds the upper limit of standard blood flow on $40 \%$. During the period of spontaneous expulsive efforts, there is a significant increase of IOP (45\%), while the intraocular blood flow decreases on $42 \%$. Thus, there exist an overstrain of compensatory mechanisms, playing a significant part in cerebral circulation control and an eye hydrodynamic functions as well. Hemo- and hydrodynamic oscillations, mentioned above, doesn't have drawbacks of all kinds available in the case of emmetropia, but in the case of severe forms of myopia may cause retinal detachment. Undoubtedly, gestosis may intensify such disease conditions. Further increase of blood pressure may cause a disturbance of compensatory mechanisms of the system of blood circulation. It may lead to the grave consequences for the visual system: hemorrhage, retinal detachment among them. Suffice it just here to say, some specialist can advice to stop the period of spontaneous expulsive efforts. In other words, to start cesarean section $[20,21]$. It has been very well known quite long since that the disturbance of eye hydrodynamic takes the first place in the reasons of chorioretinal degeneration. Retinal lasercoagulation has been advised as a method of prevention of ophthalmologic complications even in the period of pregnancy. Further delivery won't be contraindicated.

\section{Conclusions}

Regular medical check-up begins from the moment of pregnancy detection (1-st screening). A general organist examination, subspecialty consultations, ophthalmologic examination have to be taken. The information about previous pregnancy is required, as well as all the complications for the organ of vision does. Findus oculi description is required. The pregnant woman is appointed for the hospitalization in case of a severe complicated stages of myopia; its progression and in the case of degenerative changes in delivery management.

Further ophthalmological examination takes place on the 28-30 week ( $2^{\text {nd }}$ screening) in the case of uncomplicated myopia of a high or medium stage and initial or moderate stages of fundus oculi changes without extragenetal complications and on the 37-38 week (3d screening) for method of delivery selection.

Pregnant women with arterial hypertension or hypotension, anemia, gestosis are considered to be in a group of high risk of complications, with certain deficits of blood flow in eye region. Abortion is indicated in the case of ineffectiveness of symptomatic therapy of gestosis and of a background disease (arterial hypertension), as well as in a state of the progression of eye disorders such as retinal hemorrhage, papilloedema, retinal detachment. The decision between abortion and early labors depends on the general patient state, gestation period, features and dynamic of fundus oculi changes. Supplemental examination can be taken in the hospital in the case of decompensation of the underlying disease, as well as obstetrical complications. Outpatient treatment can be taken in the case of early stages of gestosis, moderate anemia and gestation edema. Laser treatment is required in the case of retinal detachment during the period of pregnancy with complications of pregnancy or in a case of the lesion focuses of vitreochoriodistrophy (beyond period of pregnancy and type of refraction). Laser is considered to be the most effective and safe way of prevention of retinal detachment. In such a case labors through maternal passages are indicated (if the lasercoagulation was made 2 month before the period of labors).

\section{Statement of Competing Interests}

Authors have no competing interests.

\section{References}

[1] Serov V.N, Markin S.A., Lubnin A.Yu. Eclampsia. M.: Open Company «MIA», 2002. 462 p.

[2] Petrova O.Yu. Features of the clinical course of myopia with physiological pregnancy, gestosis and the postpartum period: Authoref. dis.. .. kand. med. science. M., 2004. 22 p.

[3] Akhvlediani K.N., Mazurskaya N.M., Logutova L.S. et al. Optimization of management policy for pregnancy and labors in patients with moderate and severe myopia // Rus. Lead obstetrician-gynecologist. 2002. 1. 59-61.

[4] Douglas K.A., Redman C.W.G. Eclampsia in the United Kingdom // British Medical Journal. 1994. 309. 1395-1400.

[5] Irgens H.U., Reisaeter L., Irgens L.M., Lie R.T. Long term mortality of mothers and fathers after pre-eclampsia: population based cohort study // British Medical Journal. 2001. 323. 12131217. 
[6] Repina M.A. Gestosis as a cause of maternal mortality // Obstetric. and women's diseases. 2000. XLIX (3). 11-18.

[7] Granatovich N.N. Retrospective analysis of maternal mortality in the gestosis // The problems of pregnancy. 2002. 5. 21-23.

[8] Ventskovsky B.M., Hodak A.A. Late toxicity (gestosis) pregnant // Urgent obstetrics. Kiev: Health, 1994. 164-195.

[9] Lopez-Jaramillo P., Casas J.P., Serrano N. Preeclampsia: from epidemiological observations to molecular mechanisms // Braz. J. Med. Biol. Res. 2001. 34. (10). 1227-1235.

[10] Radzinsky V.E., Smalko P.J. Biochemistry of placental insufficiency. M.: RUDN, 2001. 273 p.

[11] Freidman S.A., Taylor R.N., Roberts J.M. Pathophysiology of preeclampsia: hypertension and pregnancy // Clinics in Perinatology. 1991. V.18. 661-682.

[12] De Jong C.L.D., Dekker G.A., Sibai B.M. The renin-angiotensinaldesterone system in pre-eclampsia. Hypertension and pregnancy // Clinics in Perinatology. 1991. V.18. 683-711.

[13] Roberts J.M., Redman C.W. Pre-eclampsia: more than pregnancyinduced hypertension // Lancet 1993. 341. 55-57.

[14] Aligadgieva L.G., Neroev V.V., Sarugina O.I. et al. Reooftalmografii role in the evaluation of eye hemodynamics in pregnant women with myopia. // Annals of Ophthalmology. 2008. 2. $42-43$.
[15] Kharb S. Total free radical trapping antioxidant potential in preeclampsia // Int. J. Gynaecol. Obstet. 2000. V. 69. 23-26.

[16] Shaamash A.H., Elsonosy E.D., Zakhari M.M. et al. Placental nitric acid synthase (NOS) activity and nitric oxide (NO) production in normal pregnancy, pre-eclampsia and eclampsia // Int. J. Gynaecol. Obstet. 2001. V.172. 127-133.

[17] Dekker G.A., Sibai B.M. Etiology and pathogenesis of preeclampsia: current concepts // Am. J. Obstet. Gynecol. - 1999. V.181 (4). 1036-1037.

[18] Akhvlediani K.N., Logutova L.S., Travkin A.G. et al. The tactics of management and delivery in pregnant women with myopia // Rus. lead. obstetrician gynecologist. 2005. 4. 57-62.

[19] Travkin A.G., Logutova L.S., Akhvlediani K.N. et al. Features of delivery with gestosis pregnant with myopia // Annals of Ophthalmology. 2007. 4. 26-29.

[20] Rocheva S.L. The choice of method of delivery in women with varying degrees of myopia // Annals of Ophthalmology. 2006. 3. 47-51.

[21] Rocheva S.L. Controversial aspects tactic of pregnancy and childbirth in women with myopia // Actual problems of Ophthalmology: Proceedings of the All-Russian Scientific Conference of Young Scientists: Collection of the science works / Edited by Kh.P. Takhchidi. M., 2006. 309-311. 